\title{
A Theory of Affect Perception
}

Edoardo Zamuner

\begin{abstract}
What do we see when we look at someone's expression of fear? I argue that one of the things that we see is fear itself. I support this view by developing a theory of affect perception. The theory involves two claims. One is that expressions are patterns of facial changes that carry information about affects. The other is that the visual system extracts and processes such information. In particular, I argue that the visual system functions to detect the affects of others when they are expressed in the face. I develop my theory by drawing on empirical data from psychology and brain science. Finally, I outline a theory of the semantics of affect perception.
\end{abstract}

\section{Introduction}

Looking at facial expressions is a good way of seeing and sometimes knowing what emotions or feelings others experience. Emotions and feelings, however, are not facial expressions; they are mental states. So how do we explain the fact that we can sometimes see what others feel?

I am grateful to Frank Jackson and two anonymous referees for helpful comments and suggestions.

Edoardo Zamuner, Philosophy Program, La Trobe University, Melbourne, Australia.

Email: e.zamuner@1atrobe.edu.au. 
A common answer, one that both philosophy and psychology accept, is that we have a theory of mind. On this view, we know that there is a relationship between mental states and behaviour. For example, we know that smiling has something to do with happiness. As a result, when we see a person smile, we see her behaviour as something related to happiness; we see it, for example, as an expression of happiness. It is in this sense that we see what others feel.

A way of describing the interaction between knowledge - in this case, the belief that smiling has something to do with happiness - and visual perception is in terms of the interaction between cognitive and perceptual systems. On this view, a visual experience of a person smiling carries information about a number of things, including the person's mental state. It is only when the cognitive system extracts the information carried that we can see that the person has a certain mental state. This view implies a specific conception of the object of perception. Since the stimulus that reaches the visual system is the light originating from the person's behaviour, including her facial expression, it is tempting to say that the object of perception is the person's behaviour.

In what follows I develop an alternative account of the object of perception. By focusing on affective states such as emotions and feelings, I argue that what we see when we have a visual experience as of another person feeling happy is the affective state itself and not just the person's behaviour. To put it in other terms, the information about the person's affect itself is available in the stimulus that reaches the eye (namely the light originating from the person's behaviour), and the visual system functions to extract this information. 
My argument has the following structure. First, I develop an account of facial expressions as patterns of changes that carry information about the affective states that produce them. ${ }^{1}$ Second, I argue that one of the functions of the visual system is to detect these states in others, and that this function is carried out by extracting the information that expressions embody about affective states and some of their properties. I develop this part of my argument by drawing on empirical data from psychology and brain studies. In addition, I show that my account fits nicely with a simulation theory of psychological ascriptions. Finally, I provide an account for the semantics of visual experience of affective states. In particular, I define the circumstances in which an experience of affective states can be considered veridical.

\section{Expressions Carry Information about Emotions}

We have noted that looking at facial expressions is a good way of seeing what emotions and feelings others experience. This means that facial expressions are good sources of information about the affective states of others. But what, exactly, do expressions tell us about these states? The first and most obvious thing is that expressions tell us what kinds of affects others experience, or seem to experience (if we accept that sometimes others may try to deceive us). The notion of an affective state covers a range of different mental states including emotions, bodily feelings such as pains, and sensations of pleasure and displeasure. What these states have

\footnotetext{
${ }^{1}$ I will confine my discussion to visual perception of facial expressions. This is for two reasons. First, facial expressions are better understood than other kinds of expressions. Second, the empirical literature on visual processing of expressions is more abundant. Sometimes I will refer to facial expressions simply as 'expressions' and to visual perception as 'perception' or 'experience'.
} 
in common is that they affect the body of the person who has them. In other words, they are felt states. Another common feature is that the occurrence of these states often correlates with facial expressions, as is shown by the fact that simply by looking at another person's facial expression I can tell what she feels. Of course, sometimes I can be wrong about what I see but the possibility of misperception is a common feature of all forms of perception, not primarily a feature of perception of affective states.

So facial expressions tell us what kinds of emotions and feelings others experience. For example, by looking at another person's face, I can tell if she feels sad or happy, angry or bored. Expressions can also tell us something about the intensity and duration of affects. Thus, I may be able to tell if a person feels angry, slightly angry, or very angry. In some cases, I may also be able to say for how long this emotion lasts. Finally, expressions can tell us in relation to what objects and events certain emotions arise. Consider the case of someone who sees a child being attacked by a dog. If you are looking at the person's expression while she witnesses the attack, you will learn that something horrible is happening in the vicinity - something that is likely to be responsible for her surprise and alarm. In short, expressions carry information about a number of features of affective states. They can tell us what kinds of states others experience, how intense they are, how long they last, and in relation to what they occur.

Of course, it is important to bear in mind that in some cases expressions only tell us what features these states seem to have, as opposed to the features that they actually have. So, for example, an expression can tell me how long a given emotion seems to last, rather than how long it lasts in reality. This is to say that I can always be wrong about what I see in other people's expressions. (We have noted that the possibility of error is not a special problem of emotion perception; it is a common feature of all forms of perception). 
But what, exactly, is an expression? I propose the following account. Expressions are patterns of simultaneous, or nearly simultaneous, involuntary changes occurring in the face. The claim that they are patterns of changes is justified by the fact that expressions involve various physical changes in different face regions. Smiles, for example, involve changes mostly around the mouth and eyes. The second claim, the one according to which expressions involve simultaneous, or nearly simultaneous changes, is justified by the observation that expressions are the result of changes that occur roughly at the same time. This is shown by the fact that when someone expresses joy, the appearance of the whole face changes and it does so at once. Finally, the claim that expressions involve involuntary changes is justified by the fact that a voluntary expression is not really an expression, but rather a posed or contrived arrangement of facial muscles that only resembles a genuine expression.

It should be noted that empirical evidence supports the second and third claims. Studies (e.g., Schmidt, Ambadar, Cohn and Reed, 2006) have shown that when people contrive or pose their expressions, the changes involved are not simultaneous, with some changes occurring sooner than they would if the expressions were genuine. In sum, facial expressions are patterns of simultaneous changes that emotions produce in the face. This account is sufficiently general to cover the varieties of expressions that people display in their faces when they show their emotions.

We have seen that expressions tell us a number of different things about affective states. This account, however, does not explain how expressions come to carry information about these states. To explain how this happens, I will outline an account of the information-carrying character of expression. The notion of information that I have in mind is the one adopted by Fred Dretske (1981) in his information-theoretic approach to knowledge. Roughly speaking, the 
approach says that the meaning of a sign is a function of what it indicates. Thus tracks in the woods indicate or carry information about the fact that there are deer in the woods because, all things being equal, the tracks would not be there unless deer were there. In line with this approach, one may argue that expressions carry information about affective states because, as states that show or display affects, expressions would not occur if people did not experience those states.

The view that expressions carry information presupposes that there is a reliable relationship between expressions and affects. What is this relationship exactly? To answer this question we need to consider accounts of the relationship between affects and behaviour. Since affects are felt states and emotions are, on some views, paradigms of felt states, I will focus on accounts of emotions. There are at least three philosophical theories that discuss the relationship between emotions and the modifications that we observe in the body in correspondence with emotions. On these theories, expressions are just the outwardly observable parts of these modifications. One theory (e.g., Damasio, 1994) says that emotions are just patterns of changes in the body, including the face. Another (e.g., James, 1884; Prinz, 2004) says that emotions are experiences of changes in the body. Notably, this theory identifies emotions with another class of affective state, namely feelings. A third theory (e.g., Lyons, 1980) says that emotions produce (or may produce) patterns of changes in the body, including the face. Each theory thus accepts that when we observe an expression, we observe a pattern of changes that relates to an affective state. It is in virtue of this relationship that expressions carry information about affective states.

Depending on the theory one adopts, it is possible to provide a slightly more accurate account of the information-carrying character of expressions. In particular, following the first two theories, one might say that expressions carry information about affects because expressions 
are part of the changes that either constitute the affects or lead to them. Alternatively, on the third theory, one might say that expressions carry information about affects because they are part of the changes that these states produce when they affect the body. This last theory is in line with the intuition, commonly found in folk-psychology, that some mental states are causally responsible for changes in the body and behaviour.

There is another reason for thinking that expressions carry information about affective states. All three theories accept that there is a dependence or co-variation relationship between affects and expressions such that changes in expressions generally correspond to changes in the affects that they display. On the first two theories, changes in the body (including expressions) lead or may lead to changes in affects, for expressions are just part of the bodily changes that constitute or lead to those states. On the third theory, affects may produce bodily changes. As a result, changes in affects may lead to changes in the body, including expressions. In what follows I will focus on the third theory and describe expressions as patterns of changes that affective states produce in the face. It is in virtue of this dependence relationship that expressions carry information about the affective states that produce them.

Some empirical evidence might lend additional support to the view that expressions carry information about affective states. I say it might because, even though they have influenced research on emotions and emotional expressions over the past 40 years, this evidence and the theory devised to explain it are now viewed with some scepticism. In a series of seminal studies, Paul Ekman and his colleagues (Ekman, Sorenson and Friesen, 1969; Ekman and Friesen, 1971; further evidence is discussed by Izard, 1971) made the observation that, in certain circumstances, individuals from totally different cultures expressed some emotions - which they named basic emotions - through very similar, if not identical, patterns of changes in the face. To put it in 
other terms, Ekman and his colleagues observed that basic emotions have characteristic facial expressions and speculated that the correlation between basic emotions and characteristic expressions become established through natural selection and is, as a result, universal.

Recent studies (e.g., Reisenzein, Bördgen, Holtbernd and Matz, 2006) have challenged both the idea that there are basic emotions and the observation that basic emotions have characteristic expressions. Yet although problematic, Ekman's observation has the potential to lend some support to the idea that expressions carry information about affective states. Suppose that it is true that certain emotions (maybe only some basic emotions, maybe other emotions) have characteristic expressions. It follows from this that when one observes an expression that is characteristic of or peculiar to a certain emotion, it is very likely that one will acquire information about the specific emotion that produced it. As a result, a given pattern of changes in the face becomes a reliable indicator for the occurrence of a specific emotion in others. It is in this way that Ekman's observation might lend support to the view that expressions carry information about the emotions that produce them. The evidence, however, is not essential to the view. As we have seen, all is needed to justify the view is that there is a correlation between affects and patterns of changes in the body, including the face.

\section{The Visual System Functions to Detect Emotions}

In this section I will develop a theory of visual perception of emotions; I will occasionally refer to this specific type of perception as 'affect perception'. The theory says that the visual system functions to detect affects, and some of their properties, in other people's facial expressions. This 
view is inspired by a philosophical approach to auditory perception. A common view maintains that sounds are the objects of auditory perception. In a number of recent papers, Matthew Nudds (2010a, 2010b; Nudds and O'Callaghan, 2009) challenges this common view and argues that the objects of auditory perception are, in fact, the objects and events that produce the sounds. He argues that sounds carry information about their sources and that the auditory system functions to extract this information. A key process in this function is that the auditory system groups together frequency components that have been produced by the same object or event. In what follows I will develop a similar approach, with the difference that I will focus on visual perception of affects.

We have seen in the previous section that the face carries a wealth of information about the emotions and feelings that others experience. It is therefore plausible to think that a theory of affect perception will share some important aspects with a theory of face perception. Bruce and Young (1986) have developed a theoretical model for face perception and recognition. The model draws on David Marr's (1982) computational theory of vision, which subsumes three explanatory projects: (a) a theory of the computation of visual information, (b) an account of the representations and algorithms used to manipulate the information, and (c) a theory of the physiology that underlies the processing of information. Bruce and Young are essentially concerned with the first project, namely a theory of how the visual system computes information about faces. Their theory also involves three stages. The first is, in Marr's terminology, a primal sketch that describes the geometrical structure of the retinal image of the face perceived. The second is a representation of the physical properties of the face's surface. Bruce and Young believe that this stage may also be involved in the processing of information about facial expressions. The third is a structural representation of the face that allows for recognition of the 
face's identity.

A similar model can be devised for affect perception. The first stage is a representation of the geometry of the expression perceived. Given that expressions are dynamic patterns of changes, this will be a spatiotemporal representation of the changes that form a certain expression. The second stage is a representation of the physical properties of the expression. This involves information about the various different changes that the face undergoes, including changes like frowning, scowling, smiling, and the changes in complexion that we observe when a face brightens, turns pale or flushes. In line with Bruce and Young's view and given the nature of the information processed, it is plausible to think that it is at this stage that the visual system acquires information about the affect that an expression shows. The third stage consists in the identification or recognition of the specific kind of affect that is expressed in the face. This involves a comparison between the representation produced in the previous stages and information stored in the form of beliefs. In what follows I will only focus on the first two stages and leave aside the third one.

How, exactly, does the visual system acquire information about affects? To answer this question we need to consider the complexity of the stimulus that reaches the eye when we look at facial expressions and how the visual system processes the stimulus. I will clarify this point by considering an example from ordinary perception of physical objects. Think of what you see when you try to cross the road. A number of objects, most of them with opaque surfaces, move before your eyes in rapid succession. The stimulus is therefore a spatiotemporal sequence of opaque surfaces in motion. The fact that you have a clear visual experience of the traffic on the road - an experience that represents the cars, the bicycles, and the pedestrians - means that the visual system is able to organise and manipulate the stimulus in such a way that it acquires 
information about which surfaces are cars, which ones are bicycles, and which are pedestrians.

Similar considerations apply to the stimulus that reaches the eyes when you look at someone's facial expression. The visual system faces two difficulties in this case. One is to distinguish the face - the specific one that you are interested in - from the background. This background, depending on the situation, may consist of a number of different opaque surfaces, some of which are static while others are dynamic. The surfaces correspond to the different physical objects - people, furniture, trees, etc. - in your visual field. It is from this background that the visual system needs to distinguish the specific facial expression at which you are looking. The other difficulty is to identify the expression from the sequence of physical changes that the visual system detects when you look at someone's face. This involves distinguishing what is from what is not an expression.

To understand the kind of process that the visual system needs to carry out in order to deal with the stimulus, it may be helpful to consider another example from ordinary visual perception. Suppose that you are looking at the night sky. All you see are the stars against the black background. At some point, however, you notice that four little dots above the horizon are not stars but the lights of an airplane in motion. How do you distinguish the airplane from the stars, given that they all have the same appearance? The answer is that the four lights move in the sky at the same speed. It is by virtue of this property that you are able to distinguish the four lights and, consequently, the airplane from the stars in the background. This example shows that the visual system detects the airplane because it groups together the four moving lights. This is a visual process by which the visual system organises the incoming stimulus - namely a collection of random bright dots against a black background - and subsequently acquires information about the airplane. 
A similar process is likely to occur with facial expressions. The visual system needs to organise the incoming stimulus and produce representations of the objects and events (or, as in this case, the affects) about which the stimulus carries information. To appreciate the complexity of the stimulus that reaches the eyes when we see other people's expressions, we need to consider how expressions change in ordinary interpersonal exchanges. Take a situation where you visually interact with and thus look at a number of people. This could be a classroom interaction, for example. The students before your eyes constantly move their faces; they blink, they bite or moisten their lips, they chew gum, they touch their faces, they yawn, they look around, and so on. To put it in other terms, you observe a number of different physical changes occurring on the students' faces. As in the airplane example, the stimulus that reaches your eyes is complex and quite ambiguous. How do you distinguish changes that are expressions from changes that are not? In other words, how do you make sense of the complex sequence of changes that you observe and parse it into meaningful units, namely expressions? The answer, I maintain, is that the visual system does most of the work - and that it does so by grouping together those changes that occur at the same time.

But how does this grouping process acquire information about the affective states that are responsible for the changes that occur on people's faces? The answer draws on the account of facial expressions that I developed in the previous section. We have seen that expressions of affects are patterns of simultaneous, or nearly simultaneous, physical changes that occur in the face. More precisely, an expression of affect is a pattern of changes that occur at the same time. It follows from this that by grouping together changes that occur at the same time, the visual system groups together changes that have been produced by the same affect. As a result, the visual system manipulates the incoming stimuli in such a way that it organises them in relation to 
the affect or affects responsible for the patterns of changes.

It should be noted that grouping is a common visual process that works according to a number of law-like principles (Bruce and Green, 1985). For example, the visual system groups together aspects of a visual scene because they are spatially close together, or because they appear similar, or because they have a certain relation to their background. Although grouping works particularly well with static elements, there are principles that govern the grouping of moving elements. One of these is known as the principle of the common fate, according to which things that appear to move together are grouped together. Another is the principle of temporal synchrony, according to which elements that undergo simultaneous and coherent changes (e.g., having synchronous onset and offset) are grouped together (Lee and Blake, 1999; Handel, 2006).

Some early studies have shown that these principles may be involved in the capacity to acquire information about affective states from simple patterns of facial movements. This is particularly clear in an experiment conducted by John Bassili (1979). The experiment involved a simple recognition task where video recordings of actors mimicking facial expressions of emotions were shown to the participants. The interest of the experiment consists in the stimulus shown. Before making the recordings, the actors had black makeup applied to their faces, while white spots were placed on some key points such as the forehead and cheekbones. The recordings were then played back so that only the white spots were visible. The results demonstrated that changing displays of happiness, sadness, fear, surprise, anger and disgust were recognized more accurately than static displays of the white spots. This indicated that the ability to recognize emotions depends, at least in part, on an early visual process that groups together patterns of simultaneous changes.

What I have so far observed suggests that grouping is likely to be responsible for the 
capacity to detect what affective states others experience. As a process that combines simultaneous changes, grouping is also likely to be responsible for the capacity to detect the seeming duration of an affect. It is more difficult to picture how grouping might determine the capacity to perceive the intensity of an affect. Such a process is likely to be driven by previously stored information. This would be a top-down operation in which a sequence of changes is grouped in a certain way because it fits into a perceptual pattern that the visual system recognises as likely to have been produced by an affect with a certain intensity.

A possible objection to my approach is that, as is shown by a number of psychological studies, people can detect affective states in others even when they are looking at pictures of expressions. One might thus conclude that grouping is not essential to the capacity to detect what affects others experience and that my theory is therefore mistaken. In reply two points can be made. First, although it is true that observers are able to detect affects from pictures, it is also true that judgments based on viewing pictures are often less accurate than the judgments based on the observation of moving expressions. This is confirmed by a number of studies, including Bassili's experiment above and Paul Ekman's early studies on affect recognition. It is plausible to think that still expressions, such as those we see in pictures for example, are less informative than moving expressions. In addition, it should be noted that it is becoming increasingly popular in psychological experiments to use moving expressions instead of still ones. The second observation is that the capacity to detect affects from still expressions is likely to depend upon and derive from the capacity to detect affects from ordinary moving expressions.

A system that processes the stimulus in the way I have described is a system that restores the information that the stimulus embodies. Since such information is about affective states, a system that works this way is a system that functions to acquire information about affective 
states. It makes good sense, from an evolutionary point of view, that the visual system has evolved to function this way. Affects are mental states that impact directly upon behaviour. Anger, for example, may lead to violent behaviour. If a creature has the ability to detect the occurrence of this emotion in others, it consequently stands better chances of avoiding dangerous situations, situations in which other creatures may attack and harm it. It follows from this that the capacity to detect affects has a clear survival value.

It is in terms of this function that we may explain an important feature of visual experiences of affective states, namely the fact that both the expression and the affective state feature in the representational content of a visual experience of what someone feels. Consider the following example. By looking at a person's facial expression, you see that she feels happy. This requires you to see her facial expression in the first place. Of course, you may not be aware of the fact that you are seeing the expression, but the fact remains that, in order to see that the person feels happy, you need to see her expression. When you have visual experience as of another person feeling happy, it is in the facial expression that you see the emotion. This suggests that whenever we see what another person feels, and the state is expressed in the face, we cannot see the affect without, at the same time, seeing the expression. This feature of the representational content of affect perception can be viewed as resulting from the way the visual system works to recover information about affective states. One may indeed argue that both the expression and the affect feature in the representational content of experience because it is by processing the expression that the visual system acquires information about the affect. On this view, expressions can be described as stages of the process that the visual system carries out to acquire information about affects.

The result of the process that I have described is a visual representation of affective 
states. It is important to bear in mind that this representation does not recognise what kinds of emotions or feelings other people have. The task of recognising the affects is carried out in the third stage of visual processing, which involves a comparison between the representation produced in the previous stages and information stored in the form of beliefs. It is this process that allows the perceiver to identify the affective states. In order for this identification to occur, the visual system needs to extract the information that expressions carry about affects. Such information is subsequently manipulated and conceptualised in the third stage, enabling the perceiver to identify the affects. In this view, the identification of what kinds of affective states others have depends upon a more fundamental perceptual process whose function is to detect that others have those states.

\section{Underlying Physiology}

We have seen that Marr's theory of vision comprises three explanatory projects: (a) a theory of the computation of visual information, (b) an account of the representations used in the computation, and (c) a theory of the underlying physiology. In this section I will develop a model of the physiological structures that may be involved in the process that I described in the previous section. Although my model is highly speculative, it draws on empirical evidence from studies of people who have lost the capacity to visually detect what emotions and feelings others experience. I use this evidence to argue that some specific brain structures are involved in the process of acquiring information about these states. I do not claim that these are the only structures involved. 
Studies (Adolphs, Tranel, Damasio and Damasio, 1994; Adolphs, Tranel, Damasio and Damasio, 1995) have shown that acquired damage to the amygdala results in a deficit characterised by the inability to recognise fear when it is expressed in the face. Notably, the deficit does not occur when fear is expressed through other mediums, such as speech for example (Adolphs and Tranel, 1999). Initially it was thought that the deficit compromised the ability to recall stored information about the ways in which fear is expressed in the face (Adolphs, 2002). In this interpretation, the damage would affect the ability to access part of the information that is used to interpret patterns of changes in the face. In recent years, however, researchers (Adolphs, Gosselin, Buchanan, Tranel, Schyns and Damasio, 2005) have noticed that subjects with amygdala damage fail to spontaneously attend to the eye region when they are presented with facial expressions of fear, and that the problem disappears as soon as they are explicitly instructed to attend to the eye region. This simple instruction was sufficient for the subjects to recognise fear in expressions. Interestingly, when no explicit instruction was given, the subjects reverted to their previous practice and failed to attend to the eye region.

One may speculate that the amygdala plays a key role in directing attention to specific features of expressions - features that carry a particular weight in terms of the information they provide about affective states. This hypothesis is confirmed by evidence that changes in the eye region convey most information about affects (Ellis, 1975; Thompson, 1980). Subjects with amygdala damage fail to attend to the eye region and, as a result, fail to acquire the information that changes around the eyes provide. This means that in producing a visual experience of another person's face and expression, the visual system of a person with this sort of damage fails to acquire part of the information available in the face - a part that, as the evidence shows, carries crucial information about affects. This may explain why subjects with amygdala damage 
have difficulties in detecting fear but retain knowledge and understanding of this emotion - that is, their theory of mind (and, in particular, what the theory says about fear) is relatively intact.

What sort of visual experiences will one have in this case? The evidence indicates that subjects with amygdala damage have normal vision. This means that the deficit does not affect their visual experience by making it blurry, out of focus, or phenomenally inaccurate in the way short sight or colour blindness does. One may speculate that, in this case, a visual experience of another person's affect will be an ordinary experience of her facial expression, with the difference that, as a result of the damage, the experience will carry little or no information about what goes on in the eye region. Consequently, it will carry little information about the affect or affects expressed in the face.

This sort of experience is not as uncommon as it may seem. Consider the case of looking at a person who is wearing large sunglasses. Since the glasses obscure the whole eye region, most of the information about the person's affective state is not available to you. This may make it difficult for you to say what emotion or feeling the person is expressing. It is possible that when the visual system fails to direct attention to the eye region, the resulting visual experience lacks information in the same way as a visual experience of someone wearing large sunglasses does.

What I have observed so far shows that the capacity to see what others feel is the result of a specific visual process. The evidence I have examined suggests that the amygdala is a key component in this process and that it works by directing the attentional focus to aspects of facial 
expressions that carry a great deal of information about affects. ${ }^{2}$ Although the amygdala does not seem to play any role in the grouping process that I described earlier, one can assume that if an observer does not attend to the eye region, then the visual system will group together only a limited number of changes and, as a result, will produce experiences that do not carry much information about the affects that others experience. It makes good sense to think that experiences like these will not provide the observer with sufficient evidence for deducing what others feel.

\section{Simulation Theory}

The approach I have developed so far draws on the observation that looking at people's expressions is a good way of seeing and sometimes knowing what they feel. The explanation I have provided for this observation is that the visual system functions to detect affects in others. As such, my theory has the potential to contribute to our understanding of the problem of other minds and, in particular, to the question of how we make attributions of mental states (and affects in particular) to others. There are two main accounts of how we make psychological attributions to others. They are the theory theory (e.g., Lewis, 1972; Premack and Woodruff, 1978; Gopnik and Wellman, 1992; Gopnik and Meltzoff, 1997) and the simulation theory (e.g.,

\footnotetext{
${ }^{2}$ Of course, it is possible that other brain structures or networks are involved in the process. For example, there is evidence (Sprengelmeyer, Young, Calder, Karnat, Lange, Homberg, Perrett and Rowland, 1996) that subjects with Huntington's disease have difficulties detecting disgust when it is expressed in the face.
} 
Gordon, 1986; Heal, 1986; Humphrey, 1984; Goldman, 1989; Harris, 1992). The theory theory says that we have a theory of mind that we use to explain other people's behaviour and overt bodily changes (the sort of changes involved in expressions). This theory consists of beliefs and psychological laws that describe the relationship between mental states and behaviour. We need to know this theory, implicitly or otherwise, in order to make attributions of mental states.

The simulation theory says that we are able to explain other people's behaviour, and thus make psychological attributions, because, as observers, we can imaginatively put ourselves in other people's place; that is, we can imagine what they think and feel in certain situations. This provides us with an understanding, from the inside, of what goes on in other people's minds. A stronger version of the same theory (Goldman, 2006) says that we do something more than simply imagine what others think and feel; we actually form the same mental states in ourselves and thus we understand, from our point of view, what states they are in or experience. This view maintains that when we see someone with a certain mental state - pain, for example - we undergo a pattern of modifications in our body, including the brain, that is similar to the modifications that we normally undergo when we are in pain. The appeal of the simulation theory is that it does not require an observer to know a theory or psychological laws. All she needs is the ability to imagine or, according to the strong version of the theory, to simulate the mental states that she observes in others. It is to this approach that my theory of affect perception can make a contribution. I will explain how.

In the previous section I described a process that extracts information about the kind of mental state that another person has or experiences in a purely visual way. This is to say that the information can be acquired even when the observer knows no theory of mind or psychological laws. In line with the simulation theory, we can suppose that, in certain situations, this specific 
piece of information may trigger a simulation process that mimics the kind of state or states (and affects in particular) that the process detects in others. Depending on the type of simulation theory that we embrace, we can then suppose that the simulation process consists of mere imaging or of a state that is similar in kind to the one observed in others. This supposition, in turn, provides the ground for a psychological ascription of the right kind. On this view, my approach offers an account of what happens in the early stages of those simulation processes that, according to the simulation theory, are responsible for our ability to understand what others feel.

This account has two advantages. First, the approach gives us a way of explaining how visual information can lead to psychological ascriptions without assuming that the process is driven by knowledge. This has the potential to explain how observers who do not have knowledge of a theory of mind or psychological laws - such as children, for example - can make sense of the behaviour they observe in others and often make correct psychological attributions. Second, my approach does justice to the observation that the ability to understand what others feel seems to have something to do with the fact that, in some situations, we experience in ourselves the same affects that others seem to us to experience and we do so in response to the behaviour we observe in them.

\section{Correct Conditions for Visual Experiences of Emotion}

Like any other experience, a visual perception of another person's affect may be accurate or inaccurate. It is when the experience is accurate that the perceiver stands better chances of 
knowing, as opposed to merely believing, what another person feels. But when, exactly, do we describe this kind of visual experience as being accurate? To put it in other terms, what conditions does an experience of another person's affect need to meet in order to count as accurate?

A first condition is that the visual system needs to be working properly. If the system does not work properly, it produces experiences that are inaccurate. This is clear in the case of brain lesions that prevent the system from acquiring information from the eye region. Suppose that we show a subject with amygdala damage an expression of fear. If, in forming the experience, the visual system fails to carry out its function and thus detect fear, the experience will lack information about the emotion expressed. A visual experience of someone expressing anger that does not carry information about anger is an experience that lacks a specific piece of information -information that, in my account, the visual system functions to detect. In short, one condition for accurate visual experiences of other people's affects is that the visual system works properly.

Is there any situation in which the visual system works properly and, nonetheless, produces visual experiences of other people's affects that are inaccurate? I can think of at least one scenario. This is when people pretend to be sad because they want to deceive us or because they are acting. Posed or contrived facial expressions are patterns of changes that aim to resemble the changes that emotions and feelings normally produce in the face. The difference is that unlike genuine expressions, these kinds of changes are not actually produced by affects. When these kinds of patterns are the stimulus that reaches the visual system, the experiences that result are inaccurate because there are no affects of which the experiences are forms of perception. 
But why, one might ask, does the visual system process these patterns even though they are not actually produced by affects? As we noted in section two, posed or contrived expressions involve patterns of changes that are not as simultaneous as those involved in genuine expressions, for the onset and offset of some changes is slightly delayed. This may explain why these expressions often look unnatural or, indeed, contrived without looking significantly different from genuine ones. It is plausible to think that, in some cases, the late onset or offset of the changes is difficult to detect. As a result, the visual system may process these specific kinds of changes in the same way as it processes the changes that affects normally produce in the face.

This means that there are at least two kinds of patterns that can produce a visual experience as of someone experiencing an affect - such as sadness for example. One is a pattern that is actually produced by sadness. The other is a pattern that is not produced by sadness but that closely resembles the sort of pattern that sadness normally produces. If, on a given occasion, the visual system processes the latter pattern in the same way as it processes the former, the result will be an experience that, in spite of representing someone's sadness, is inaccurate because there is no sadness of which the experience is a perception.

(Word count: 7,846 words)

Philosophy Program

La Trobe University 


\section{References}

Adolphs, R. 2002: Neural systems for recognizing emotion. Current Opinion in Neurobiology, $12,169-177$.

Adolphs, R., Gosselin, F., Buchanan, T.W., Tranel, D., Schyns, P. and Damasio, A.R. 2005: A mechanism for impaired fear recognition after amygdala damage. Nature, 433, 68-72.

Adolphs, R. and Tranel, T.W. 1999: Intact recognition of emotional prosody following amygdala damage. Neuropsychologia, 37, 1285-1292.

Adolphs, R., Tranel, D., Damasio, H. and Damasio, A. 1994: Impaired recognition of emotion in facial expressions following bilateral damage to the human amygdala. Nature, 372, 669672.

Adolphs, R., Tranel, D., Damasio, H. and Damasio, A. 1995: Fear and the human amygdala. Journal of Neuroscience, 15, 5879-5891.

Bassili, J.N. 1979: Emotion recognition: the role of facial movement and the relative importance of upper and lower areas of the face. Journal of Personality and Social Psychology, 37, 2049-2058.

Bruce, V. and Green, P.R. 1985: Visual Perception. Physiology, Psychology and Ecology. London: Lawrence Erlbaum Associates Ltd. Publishers.

Bruce, V. and Young, A. 1986: Understanding face recognition. British Journal of Psychology, $77,305-327$

Damasio, A.R. 1994: Descartes' Error. Emotion, Reason and the Human Brain. New York: G.P. Putnam's Sons. 
Dretske, F. 1981: Knowledge and the Flow of Information. Cambridge, MA: MIT Press.

Ekman, P. and Friesen, W.V. 1971: Constants across cultures in the face and emotion. Journal of Personality and Social Psychology, 17, 124-129.

Ekman, P., Sorenson, E.R. and Friesen, W.V. 1969: Pan-cultural elements in facial displays of emotions. Science, 164, 86-88.

Ellis, H.D. 1975: Recognizing faces. British Journal of Psychology, 66, 409-426.

Goldman, A.I. 1989: Interpretation psychologized. Mind and Language, 4, 161-185.

Goldman, A.I. 2006: Simulating Minds. The Philosophy, Psychology, and Neuroscience of Mindreading. Oxford: Oxford University Press.

Gopnik, A. and Meltzoff, A.N. 1997: Words, Thoughts, and Theories. Cambridge, MA: Bradford, MIT Press.

Gopnik, A. and Wellman, H.M. 1992: Why the child's theory of mind really is a theory. Mind and Language, 7, 145-171.

Gordon, R.M. 1986: Folk psychology as simulation. Mind and Language, 1, 158-171.

Handel, S. 2006: Perceptual Coherence. Hearing and Seeing. Oxford: Oxford University Press.

Harris, P.L. 1992: From simulation to folk psychology: the case for development. Mind and Language, 7, 120-144

Heal, J. 1986: Replication and functionalism. In J. Butterfield (ed.), Language, Mind, and Logic. Cambridge: Cambridge University Press.

Humphrey, N. 1984: Consciousness Regained. Chapters in the Development of Mind. Oxford University Press.

Izard, C.E. 1971: The Face of Emotion. New York: Appleton-Century-Crofts. 
James, W. 1884: What is an emotion? Mind, 9, 188-205.

Lee, S.H. and Blake, R. 1990: Visual form created solely from temporal structure. Science, 284, $1165-1168$

Lewis, D. 1972: Psychophysical and theoretical identifications. Australasian Journal of Philosophy, 50, 249-258.

Lyons, W. 1980: Emotion. Cambridge: Cambridge University Press.

Marr, D. 1982: Vision. A Computational Investigation into the Human Representation and Processing of Visual Information. New York: Freeman.

Nudds, M. 2010a: What sounds are. Oxford Studies in Metaphysics, 5, 279-302.

Nudds, M. 2010b: What are auditory objects? Review of Philosophy and Psychology, 1, 105122.

Nudds, M. and O'Callaghan, C. (eds), 2009: Sounds and Perception. New Philosophical Essays. Oxford: Oxford University Press.

Premack, D. and Woodruff, G. 1978: Does the chimpanzee have a theory of mind? Behavioral and Brain Sciences, 4, 515-629.

Prinz, J. 2004: Gut Reactions. A Perceptual Theory of Emotion. Oxford: Oxford University Press.

Reisenzein, R., Bördgen, S., Holtbernd, T. and Matz, D. 2006: Evidence for strong dissociation between emotion and facial displays: the case of surprise. Journal of Personality and Social Psychology, 91, 295-315.

Schmidt, K.L., Ambadar, Z., Cohn, J.F. and Reed, L.I. 2006: Movement differences between deliberate and spontaneous facial expressions: zygomaticus major action in smiling. 
Journal of Nonverbal Behavior, 30, 37-52.

Sprengelmeyer, R., Young, A.W., Calder, A.J., Karnat, A., Lange, H.W., Hömberg, V., Perrett, D.I., and Rowland, D. 1996: Loss of disgust: perception of faces and emotions in Huntington's disease. Brain, 119, 1647-1665. 